\title{
DESCONSTRUINDO A AMARELINHA: UM JOGO DIDÁTICO NO ENSINO DA ISOMERIA PLANA
}

\section{DESCONSTRUING THE YELLOW: A TEACHING GAME IN THE EDUCATION OF THE FLAT ISOMERIA}

\author{
Gabriela Rejane Silva de Medeiros ${ }^{1}$; Danielly Francielly dos Santos Silva ${ }^{2}$; Natália Kelly da \\ Silva Araújo ${ }^{3}$; Ayrton Matheus da Silva Nascimento ${ }^{4}$; Kilma da Silva Lima Viana ${ }^{5}$
}

\section{RESUMO}

Quando se fala do Ensino de Química, é notório observar um grande desinteresse por parte dos estudantes durante as aulas. Esse fato pode ser atribuído aos métodos tradicionais de ensino que, aliados a conteúdos complexos, tornam as aulas monótonas e desestimulantes. Nesse contexto os jogos didáticos vêm ganhando espaço nas salas de aula, onde professores utilizam essa ferramenta como objetivo de complementar seus recursos educativos e tornar a aula mais dinâmica e interativa, não apenas como uma forma de transmissão de conhecimento, mas também como compartilhamento deste. O presente trabalho tem por objetivo descrever o desenvolvimento, aplicação e avaliação com a utilização do Ciclo da Experiência Kellyana (CEK) do jogo didático "Desconstruindo a Amarelinha Isomérica" em uma escola da Rede Pública do Município de Vitória de Santo Antão - Pernambuco, na qual é parceira do Programa Despertando Vocações para Licenciaturas (PDVL - IFPE), com estudantes do $3^{\circ}$ Ano do Ensino Médio. A atividade foi desenvolvida pelos membros do Grupo de Trabalho (GT) de Jogos Didáticos no Ensino de Química do PDVL do Instituto Federal de Pernambuco - Campus Vitória de Santo Antão, constatou-se que o jogo didático possibilitou uma melhor compreensão acerca do conteúdo abordado na intervenção. Os jogos enquanto função educativa propicia ao aluno uma melhor compreensão do conteúdo, além de contribuir no desenvolvimento social e intelectual dos alunos. Por se tratar de uma atividade geralmente coletiva, incentiva à interação entre os alunos, provocando discussões que podem sanar dúvidas referentes ao conteúdo ou até mesmo o surgimento de outras, que poderão ser discutidas e esclarecidas durante a atividade.

Palavras-Chave: Ensino secundarista, Química Orgânica, Aprendizagem.

\begin{abstract}
When talking about Chemistry Teaching, it is notorious to observe a great lack of interest on the part of the students during the classes. This fact can be attributed to the traditional methods of teaching that, together with complex contents, make the classes monotonous and discouraging. In this context, didactic games are gaining space in classrooms, where teachers use this tool to complement their educational resources and make the classroom more dynamic and interactive, not only as a way of transmitting knowledge, but also as a sharing of knowledge. The present work aims to describe the development, application and evaluation using the Kellyana Experience Cycle (CEK) of the didactic game "Deconstructing the Yellowling Isomeric" in a school of the Public Network of the Municipality of Vitória de Santo Antão - Pernambuco, in the which is a partner of the Awakening Vocations for

\footnotetext{
${ }^{1}$ Licencianda em Química pelo IFPE - Campus Vitória, Membro do GT de Jogos Didáticos do PDVL/IFPE - Email: medeirosgabriela32@yahoo.com.br

${ }^{2}$ Licencianda em Química pelo IFPE - Campus Vitória, Membro do GT de Jogos Didáticos do PDVL/IFPE - Email: danysantos023@ outlook.com

${ }^{3}$ Licencianda em Química pelo IFPE - Campus Vitória, Membro do GT de Jogos Didáticos do PDVL/IFPE - Email: nataliakellybs@gmail.com

${ }^{4}$ Especialista em Ensino de Química - UCAM - Prominas, ayrthon.matheus@ gmail.com

${ }^{5}$ Doutora em Ensino de Ciências, Docente do IFPE, kilma.viana@vitoria.ifpe.edu.br
} 
Undergraduate Programs (PDVL - IFPE), with students of the 3rd year of high school. The activity was developed by the members of the Working Group (WG) of Didactic Games in the Teaching of Chemistry of the PDVL of the Federal Institute of Pernambuco - Vitória Campus of Santo Antão, it was verified that the didactic game made possible a better understanding about the content approached in the intervention. The games as an educational function provide the student with a better understanding of the content, as well as contributing to the students' social and intellectual development. Because it is a generally collective activity, it encourages interaction among students, provoking discussions that can solve doubts about content or even the emergence of others, which can be discussed and clarified during the activity.

Keywords: Secondary Education, Organic Chemistry, Learning.

\section{INTRODUÇÃO}

$\mathrm{Na}$ atualidade o Ensino de Química tem sido um desafio para educadores, principalmente pela forma mecânica como é abordado os conteúdos relacionados com o Ensino Médio, que tem se mostrado insuficiente perto das novas tecnologias que dispomos. Sendo assim esses docentes têm sido levados a repensarem suas metodologias e a buscarem novas estratégias que promovam aulas mais dinâmicas, que estimulem a aprendizagem dos estudantes e dos conteúdos abordados em sala de aula (COSTA, 2013).

Segundo Krasilchik (2004), a maneira unidirecional que é lecionada uma aula tradicional, gera o desinteresse dos alunos e consequentemente um baixo rendimento escolar. Muitos alunos entram em um processo de decorar os conteúdos, os símbolo, conceitos e cálculos ao invés de tentarem aprender.Um dos recursos didáticos que tem ganhado espaço no processo de ensino aprendizagem são os jogos didáticos.

É através do lúdico que o professor pode desenvolver nos estudantes valores éticos e morais, formando assim cidadãos conscientes de sua realidade, além de proporcionar situações em que haja uma necessidade de conscientização de seus deveres e responsabilidades. O jogo ajuda o aluno na construção de novas descobertas, desenvolve e enriquece a sua personalidade, procura colocar o aluno em posição de pensar por si mesmo, colher dados, discutir ideias, emitir e testar hipóteses, sempre motivado pela identificação do problema, levando-os à aprendizagem alicerçada pelo "encantamento e pela curiosidade" (BERNARDELLI, 2004).

Além de promover o desenvolvimento de diversas habilidades os jogos ainda são uma diversão. De acordo com Kishimoto (1996): “a utilização do jogo potencializa a exploração e a construção do conhecimento, por contar com a motivação interna típica do lúdico". Por se tratar de uma atividade diferenciada em relação aos métodos utilizados no cotidiano escolar, as aulas tornam-se mais dinâmicas e atraentes e os alunos sentem-se mais motivados. 
O objetivo deste estudo é mostrar as contribuições que o jogo didático, especificamente no conteúdo de Isomeria Plana, proporcionam como uma alternativa no processo de ensino e aprendizagem nas aulas de química.

\section{FUNDAMENTAÇÃO TEÓRICA}

Quando se fala do Ensino de Química, é notório observar um grande desinteresse por parte dos estudantes durante as aulas, isso porque cada aluno tem um momento, um jeito de aprender e na maioria das vezes eles não conseguem entender os temas abordados devido à falta de contextualização entre a teoria vista em sala com o seu dia a dia. No Brasil, as práticas curriculares do Ensino de Química ainda são marcadas pela tendência de manutenção do "conteudismo" típico de uma relação de ensino transmissão-recepção, limitada à reprodução restrita do saber de posse do professor que repassa os conteúdos aos estudantes (BRASIL, 2006).

Para fugir da rotina na sala de aula ou para despertar o interesse dos alunos em determinados conteúdos é necessário que o professor busque metodologias distintas das que usualmente utiliza em sala de aula. Uma das alternativas seria ouso de jogos que são recursos didáticos educativos tendo o intuito de possibilitar e auxiliar na apresentação e ilustração de aspectos relevantes aos conteúdos, em revisão ou sínteses de conceitos importantes (SILVA, 2016).

Vários autores têm enfatizado a eficiência do jogo didático no Ensino de Química, em despertar a atenção dos alunos, a qual é baseada no aspecto lúdico, que, geralmente, produz efeito positivo no aspecto disciplinar. Diversos temas em Química podem ser explorados com o auxílio de jogos didáticos. Kishimoto (1996) defende o uso do jogo na escola, justificando que o jogo favorece o aprendizado pelo erro e estimula a exploração e resolução de problemas, pois como é livre de pressões e avaliações, cria um clima adequado para a investigação e a busca de soluções.

Ainda segundo Kishimoto (1996), o jogo, considerado um tipo de atividade lúdica, possui duas funções: a lúdica e a educativa. Elas devem estar em equilíbrio, pois se a função lúdica prevalece, não passará de um jogo e se a função educativa for predominante será apenas um material didático.

De acordo com Santana (2008), o objetivo de uma atividade lúdica não é apenas levar o aluno a memorizar mais facilmente o assunto abordado, mas sim, induzir o raciocínio, a reflexão, o pensamento e consequentemente a construção do conhecimento, promovendo a construção do conhecimento cognitivo, físico, social e psicomotor, além do desenvolvimento 
de habilidades necessárias às práticas educacionais da atualidade.

É importante ressaltar que a utilização do jogo não deve ser ao acaso, mas visto como uma das atividades dentro de uma sequência definida de aprendizagens e um meio a serem usados para se alcançar determinados objetivos educacionais (NASCIMENTO et al., 2015).

Cabrera (2007) explica que o lúdico pode ser utilizado como estratégia instrucional eficaz, pois impulsiona uma aprendizagem com significados estimulando no aprendiz uma predisposição para aprender, além de favorecer a imaginação e o simbolismo como criação de significados, que facilitam a aprendizagem. Fialho (2011) acrescenta ainda que a intensidade do poder do jogo é tão grande que a fascinação que ele exerce sobre as pessoas é imensurável.

Os jogos proporcionam ao aluno uma forma prazerosa e divertida de estudar, além de oferecer ao professor uma maneira diferente de avaliar a assimilação do alunado em relação aos conteúdos estudados, de revisar conteúdos ou como um meio mais dinâmico de fixar o conhecimento, permitindo a identificação de erros de aprendizagem (ZANON et al., 2008).

\section{METODOLOGIA}

Esta vivência foi executada com o jogo didático no conteúdo de Isomeria Plana lado a lado com o Ciclo da Experiência Kellyana (CEK), onde é dividida em 05 (cinco) etapas:

$1^{a}$ Etapa - Antecipação: Neste momento realizamos uma "Avaliação Diagnóstica" com os estudantes, onde foram levantadas algumas perguntas norteadoras e básicas relacionada aos conteúdos, nesta avaliação contém 05 (cinco) perguntas sobre o conteúdo de "Isomeria Plana", onde foi construído o jogo "Desconstruindo a Amarelinha Isomérica".

\section{P1: O que é isomeria plana?}

\section{P2: Quais são os tipos de isomeria plana?}

P3: Qual a isomeria apresentada nos compostos a seguir $\mathrm{CH}=\mathrm{CH}_{2}\left(\mathrm{CH}_{3}\right) \mathrm{CH}_{3}$ e

$$
\mathrm{CH}_{2}=\mathrm{CHCH}_{2} \mathrm{CH}^{3} \text { ? }
$$

P4:Qual o tipo de isomeria apresentada pelos compostos $\left(\mathrm{C}_{4} \mathrm{H}_{10} \mathrm{O}\right)$ representados abaixo?

$$
\begin{gathered}
\mathrm{I}-\mathrm{H}_{3} \mathrm{C}-\mathrm{CH}_{2}-\mathrm{CH}_{2}-\mathrm{CH}_{2}-\mathrm{OH} \text { (Álcool) } \\
\mathrm{II}-\mathrm{H}_{3} \mathrm{C}-\mathrm{CH}_{2}-\mathrm{O}-\mathrm{CH}_{2}-\mathrm{CH}_{3} \text { (Éter) }
\end{gathered}
$$

P5: A propanona e o propen-2-ol exemplificam que caso de isomeria plana?

$2^{\text {a }}$ Etapa - Investimento: Nesse momento foi concretizado uma explanação do conteúdo referente à temática do jogo, trabalhamos os conceitos dos cinco tipos de isomeria 
plana e como elas eram diferenciadas. Através disso, os sujeitos foram convocados a enraizarem seus conhecimentos sobre a proposição em questão.

$3^{\text {a }}$ Etapa - Encontro: Nesse momento, foi apresentado aos estudantes à realização e aplicação do jogo "Desconstruindo a Amarelinha Isomérica".

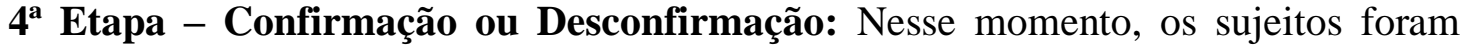
questionados novamente e foram feitas as mesmas indagações da Antecipação. Averiguando se após a vivência do encontro suas hipóteses iniciais foram confirmadas ou desconfirmadas.

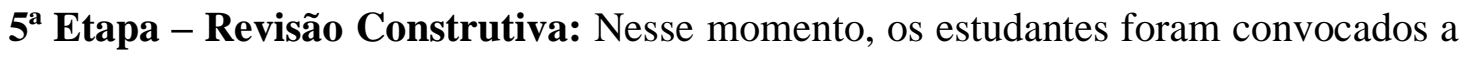
refletirem todo o processo vivenciado nas etapas anteriores, ou seja, se os estudantes tiveram alguma aprendizagem de conceito diferente ou errada, nesta etapa vai construir os novos construtos.

\section{Desconstruindo a amarelinha isomérica}

O ensino com atividades lúdicas permite ao educador criar inúmeras condições para o educando desenvolver habilidades, pois é um método atraente e interessante que proporciona aulas divertidas e dinâmicas. O jogo apresentado tem como objetivo compreender a "Isomeria Plana" de uma forma mais atrativa. O jogo é composto por uma amarelinha, onde a mesma é formada por 20 "casas" e um "céu". As casas enumeradas de 1 a 7 são referentes à cor verde, as de 8 a 14 são referentes à cor laranja e as de 15 a 20 são referentes à cor vermelha. As diferentes cores representam o nível de dificuldade de cada fase do jogo. Para estudos e elaboração desse recurso didático-pedagógico foi utilizado como fonte de consulta o estudo de Canto (2016).

Figura 01: Jogo Desconstruindo a Amarelinha Isomérica.

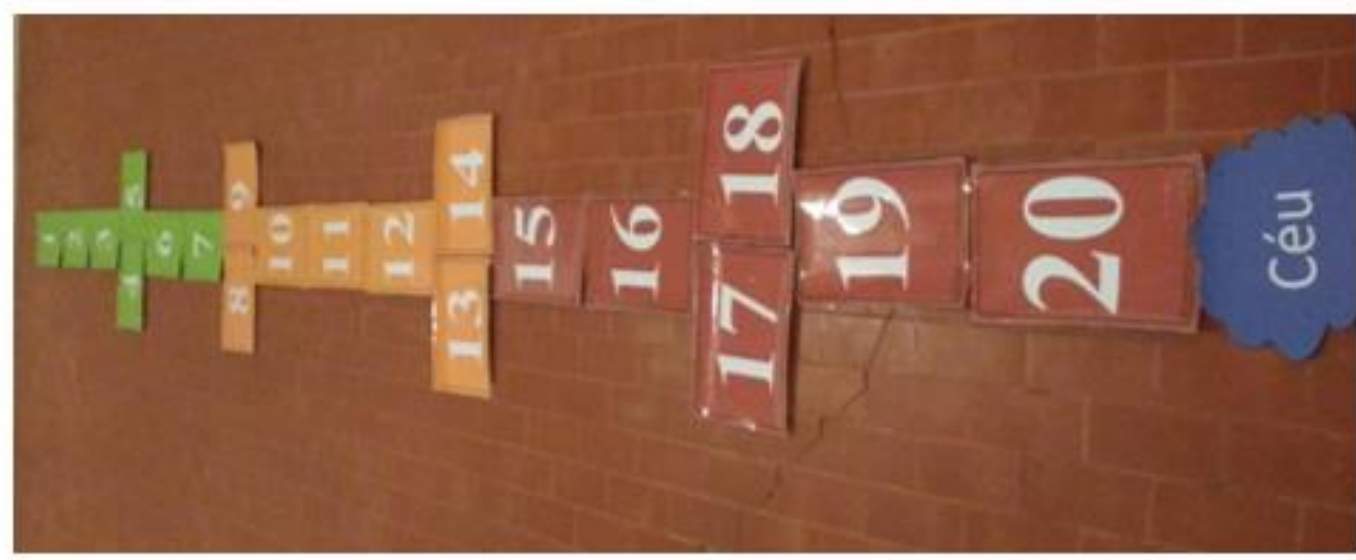

Fonte: Autor (2018). 


\section{REGRA DO JOGO}

$\checkmark$ Primeiramente a turma irá se dividir no mínimo em dois (2) grupos e no máximo quatro (4);

$\checkmark$ Depois de feita a divisão os grupos irá se subdividir em duplas e cada grupo indicará um (1) líder;

$\checkmark$ Pra dá início ao jogo os líderes tiram “zerinho ou um”;

$\checkmark$ O primeiro jogador deve jogar uma bolinha no número 1 e responder uma pergunta. Para responder a pergunta os subgrupos (duplas) precisarão chegar a uma única resposta para o grupo;

$\checkmark$ Acertando a pergunta o jogador continua pulando a amarelinha;

$\checkmark$ Errando, o líder do outro grupo deve jogar;

$\checkmark$ Voltará uma casa o jogador que pisar nas linhas do jogo;

$\checkmark$ Voltará uma casa o jogador que pisar na casa onde está a bolinha;

$\checkmark$ Só é permitido pôr um pé em cada casa. Quando há uma casa do lado da outra, podem pôr os dois pés no chão;

$\checkmark$ Quando chegar ao "céu", o jogador vira e volta pulando na mesma maneira, pegando a pedrinha quando estiver na casa 01 ;

$\checkmark$ Ganha quem terminar de pular todas as casas

\section{RESULTADOS E DISCUSSÃO}

\section{Primeira etapa do Ciclo da Experiência Kellyana (Antecipação):}

Neste instante inicia-se o ciclo, onde os estudantes foram responder as 05 (cinco) perguntas para levantarmos os conhecimentos prévios dos mesmos. As perguntas são relacionadas ao conteúdo de Isomeria Plana. O quadro 01 mostra as respostas certas (azul) e erradas (vermelha) dos grupos, em relação às 05 (cinco) perguntas vista nesta etapa. 
Quadro 01: Quadro da Primeira Etapa (Antecipação).

\begin{tabular}{|c|c|c|c|c|c|}
\hline $\begin{array}{c}\text { Grupo } \\
\text { A }\end{array}$ & PE1 & P02 & P03 & P04 & P05 \\
\hline $\begin{array}{c}\text { Grupo } \\
\text { B }\end{array}$ & CERTO & $\begin{array}{c}\text { PARCIALMENTE } \\
\text { CORRETO }\end{array}$ & ERRADO & ERRADO & CERTO \\
$\begin{array}{c}\text { Grupo } \\
\text { C CIALMENTE } \\
\text { Grupo } \\
\text { D }\end{array}$ & $\begin{array}{c}\text { PARCIALMENTE } \\
\text { CORRETO }\end{array}$ & $\begin{array}{c}\text { CARCIALM } \\
\text { CORRETO }\end{array}$ & CERTO & CERTO \\
\hline \multicolumn{7}{|c|}{ ERRADO } & $\begin{array}{c}\text { PARCIALMENTE } \\
\text { CORRETO }\end{array}$ & CERTO & ERRADO & ERRADO \\
\hline
\end{tabular}

Fonte: Autor (2018).

Figura 02: Momento da Primeira Etapa.
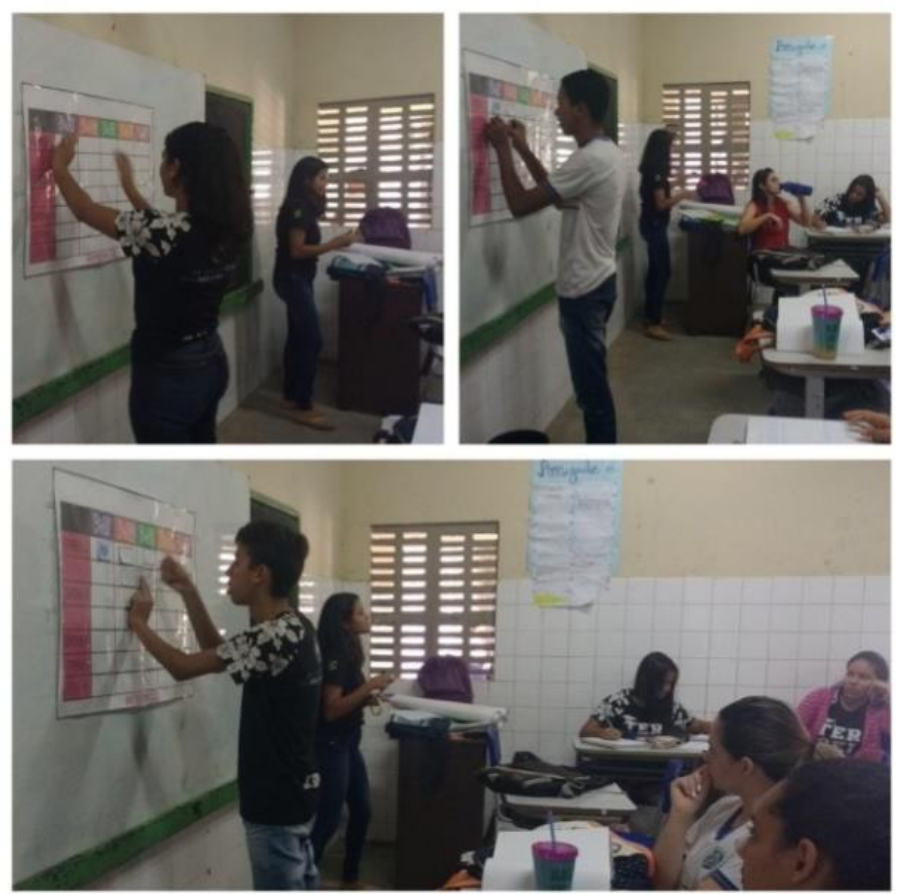

Fonte: Autor (2018).

Podemos notar que os Grupos B teve um bom desempenho em relação às perguntas feita, já o Grupo A conseguiu responder corretamente duas questões e uma questão parcialmente. E o Grupo $\mathrm{C}$ consegui responder uma questão corretamente e duas questão parcialmente. O Grupo D teve uma maior dificuldade em relação aos demais conseguindo responder apenas uma questão corretamente e uma questão parcialmente.

\section{Segunda etapa do Ciclo da Experiência Kellyana (Investimento):}

Após a vivência da etapa anterior, foi mostrada uma aula expositiva onde o estudante apresenta um papel ativo no processo, em que a voz do aluno é importante para a construção das réplicas e dos eventos vivenciados. Nesta etapa é o momento de tirar todas as dúvidas para que consiga prosseguir para próxima etapa, com isso ajudará no desenvolvimento e 
apropriação dos conceitos.

Figura 03: Momento da Segunda Etapa.

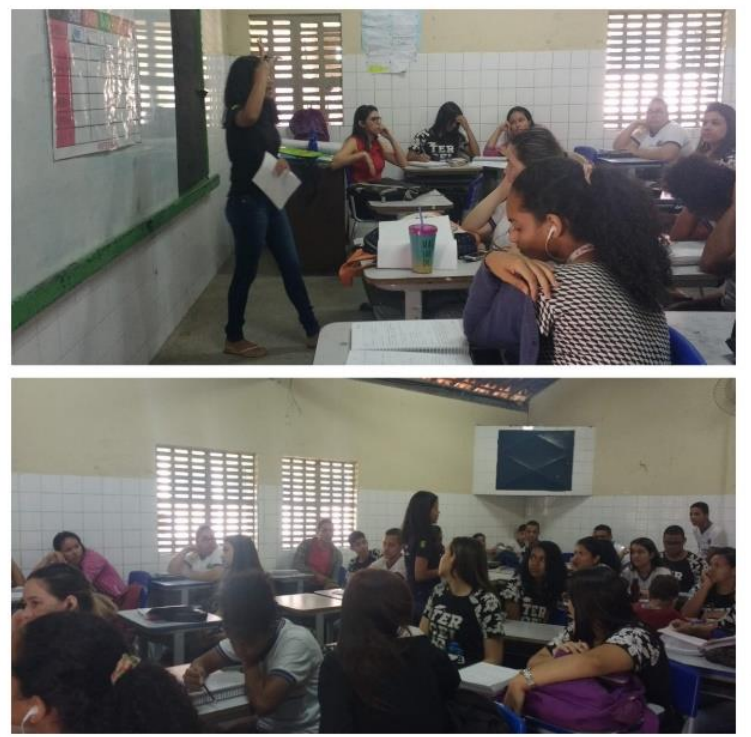

Fonte: Autor (2018).

\section{Terceira etapa do Ciclo da Experiência Kellyana (Encontro):}

É a etapa em que os alunos vivenciam o jogo chamado de "Desconstruindo a Amarelinha Isomérica", onde o objetivo é identificar os tipos de isomeria que acontece entre alguns compostos bem como os tipos de isomeria se diferenciam. Na figura abaixo mostra o momento em que os estudantes interagem entre si, comprovando uma das características que o jogo didático apresenta: a interação entre estudante-estudante e estudante-jogo, assim conseguem construir os conceitos em grupo.

Figura 04: Momento da Terceira Etapa.
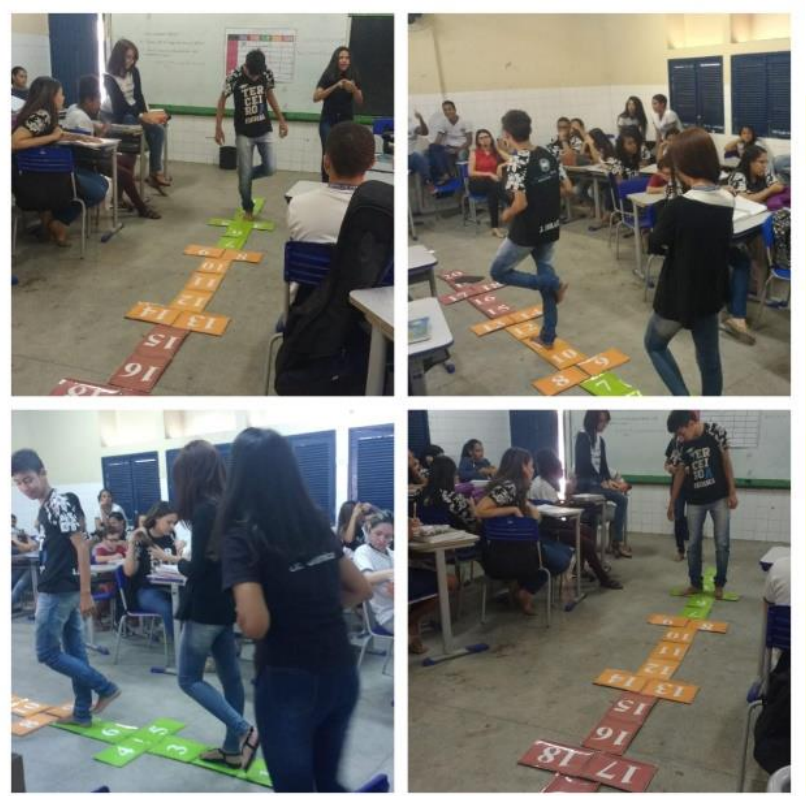

Fonte: Autor (2018).

[140] 


\section{Quarta etapa do Ciclo da Experiência (Confirmação ou Desconfirmação):}

Neste momento, os estudantes foram novamente questionados a respeito das 05 (cinco) questões da primeira etapa. Pode-se notar que todos os grupos conseguiram assimilar o conteúdo de uma forma correta o que implicou nos resultados desta etapa.

\begin{tabular}{|c|c|c|c|c|c|}
\hline \multicolumn{7}{c}{ Quadro 01: Quadro da Quarta Etapa (Confirmação ou Desconfirmação). } \\
\hline Grupo A & PE1 & PE2 & P03 & P04 & P05 \\
\hline Grupo B & CERTO & CERTO & CERTO & CERTO & CERTO \\
\hline Grupo C & CERTO & CERTO & CERTO & CERTO & CERTO \\
\hline Grupo D & CERTO & $\begin{array}{c}\text { PARCIALMENTE } \\
\text { CORRETO }\end{array}$ & CERTO & CERTO & CERTO \\
\hline \multicolumn{7}{|c|}{ CONFIRMAÇÃO OU DESCONFIRMAÇÃO } \\
\hline
\end{tabular}

Fonte: Autor (2018).

Diante as hipóteses levantadas na primeira etapa e confirmadas na quarta etapa, as respostas dos estudantes foram analisadas baseadas nos estudos de Canto (2016).

\section{Quinta etapa do Ciclo da Experiência Kellyana (Revisão Construtiva):}

$\mathrm{Na}$ última etapa, os estudantes foram questionados sobre o jogo vivenciado e pode-se perceber que o rendimento dos estudantes foi bom. Eles conseguiram compreender que o jogo didático contribuiu para a ampliação do conhecimento, além de brincarem na aula aprenderam ao mesmo tempo.

Estudante A do Grupo 01: "Foi legal aprender com os jogos, não sabia que podia aprender brincando".

Estudante D do Grupo 02: “Antes parecia que era impossível chegar ao céu, mas depois vi que tudo é possível".

Estudante D do Grupo 03: "Foi tão divertido esse aula que nem parecia que era aula de química”.

Estudante G do Grupo 04: "Com o jogo eu consegui entender o que é isomeria de uma forma bem fácil’”.

\section{CONCLUSÕES}

Pode-se notar que ao final da intervenção quando foram indagados acerca da vivência do jogo para a sua aprendizagem, uma grande parte considerou ótima, pois havia reforçado o conteúdo e puderam tirar dúvidas simples. A aplicação do jogo conseguiu despertar um interesse em estudar mais um pouco a disciplina de química. Logo se percebe que o jogo como uma ferramenta didática promoveu uma animação nos estudantes em querer participar 
do jogo, motivando desta forma a eles participarem mais das aulas e se tornarem um ser ativo no processo de ensino-aprendizagem.

Pelo exposto, entendemos que os jogos deveriam merecer um espaço e tempo maior na prática pedagógica dos professores, visto que pudemos constatar a contribuição desses jogos no processo de ensino-aprendizagem, no motivar e despertar dos estudante em relação ao estudo do Ensino de Química, facilitando a construção dos conteúdos.

\section{REFERÊNCIAS}

BERNARDELLI, M. S., Encantar para ensinar - um procedimento alternativo para o ensino da química. In: Convenção Brasil Latino América, Congresso Brasileiro e encontro paranaense de psicoterapias corporais. Foz do Iguaçu. Anais. Centro Reichiano. 2004.

BRASIL. Ministério da Educação - Secretaria de Educação Média e tecnológica. Orientações Educacionais Complementares aos Parâmetros Curriculares Nacionais: Ensino Médio. Vol. 2: Ciências da Natureza, Matemáticas e suas Tecnologias. Brasília: MEC, 2006.

CABRERA, W. B. A ludicidade para o ensino médio na disciplina de biologia: Contribuições ao processo de aprendizagem em conformidade com os pressupostos teóricos da Aprendizagem Significativa. Londrina, 2007. Dissertação (Mestrado) Universidade Estadual de Londrina, 2007.

CANTO, E. L. Química na abordagem do cotidiano, v.1. $1^{\text {a }}$ ed. São Paulo: Saraiva, 2016.

COSTA, A. K. P., TARGINO, K. C. F. et al. Utilização de jogos didáticos para o ensino de química: up and down chemical. In: Anais do IX Congresso de Iniciação Científica do IFRN, Currais Novos - Rio Grande do Norte. 2013.

FIALHO, N. N. Jogos no Ensino de Química e Biologia. 2ª edição. Curitiba: IBPEX, 2011.

KELLY, G. A. A theory of personality: the psychology of personal constructs. New York: W.W. Norton, 1963.

KISHIMOTO, T. M. Jogo, Brinquedo, Brincadeira e a Educação. São Paulo: Cortez, 1996.

KRASILCHIK, M. Prática de ensino de biologia. 4. ed. São Paulo: Universidade de São Paulo, 195 p. 2004.

NASCIMENTO, A. M. S. et al. Dominós das Funções Oxigenadas: Um Jogo Didático no Conteúdo de Química Orgânica. In: 55 Congresso Brasileiro de Química, 2015, Goiânia GO. Anais do $55^{\circ}$ Congresso Brasileiro de Química, 2015.

SANTANA, E. M. Influência de atividades lúdicas na aprendizagem de conceitos químicos. In: I Seminário Nacional de Educação Profissional e Tecnologia, 2008, Belo Horizonte - MG. Anais do Seminário Nacional de Educação Profissional e Tecnologia, 2008. 
SILVA, L. R. B. et al. Na evolução dos modelos atômicos: Um jogo didático no conteúdo de Química Geral. In: Anais do $14^{\circ}$ Congresso Internacional de Tecnologia na Educação Brasil. Recife, 2016.

ZANON, D. A. V. et al. Jogo didático Ludo Químico para o ensino de nomenclatura dos compostos orgânicos: projeto, produção, aplicação e avaliação. Ciências \& Cognição, v. 13, n. 1, p. 72-81, 2008. 